\title{
Battlefield Teleradiology
}

\author{
Daniel W. Bess ${ }^{1}$ Eric A. Roberge ${ }^{1}$
}

Published online: 7 July 2016

(C) Springer International Publishing AG 2016

\begin{abstract}
Purpose of Review Teleradiology is the transmission of diagnostic images from one location to another for the purpose of interpretation. The presence and impact of teleradiology have increased as the technology of computers, electronic picture archiving, and telecommunications have matured. In the context of the military, CT scanners are being deployed as close as possible to the point of injury, and the role of teleradiology has expanded.

Recent Findings While the integration of teleradiology services into mature healthcare environments is almost transparent to the average clinician, there are significant requirements that may be unknown to the non-radiologist.

Summary The role of teleradiology on the battlefield deserves particular attention from military planners and non-radiologist physicians involved in the resourcing and implementation of radiology services on the frontlines.
\end{abstract}

This article is part of the Topical Collection on The Military Perspective

The views expressed are those of the author(s) and do not reflect the official policy of the Department of the Army, the Department of Defense or the U.S. Government.

Eric A. Roberge

Eric.A.Roberge.mil@mail.mil

Daniel W. Bess

Daniel.W.Bess.mil@mail.mil

1 Department of Radiology, Madigan Army Medical Center, 9040A Jackson Ave, ATTN: MCHJ-CLR-D, Tacoma, WA 98431-1100, USA
Keywords Battlefield teleradiology $\cdot$ Military teleradiology · Military radiology $\cdot$ Battlefield computed tomography . Military medicine $\cdot$ Telemedicine

\section{Introduction}

Battlefield teleradiology has been utilized by the US military since the first Gulf War Operations Desert Shield and Desert Storm (1990-1991) when feasibility studies demonstrated its potential. Since then, its growth has paralleled the growth of teleradiology seen in the civilian sector where it serves a similar need. Teleradiology offers more flexible staffing options for professional radiology coverage in low-density rural areas or the austere battlefield, where small hospitals struggle to maintain adequate subspecialty coverage. Cross-sectional imaging, particularly CT, has played an increasingly vital role in the evaluation, management, and disposition of trauma and emergency patients. An exponential increase in volume and complexity of these studies has resulted in the need to maintain around-the-clock radiologist coverage for civilian emergency departments in the US since the 1980s and likewise in forward-deployed combat hospitals since the 1990s. Teleradiology has afforded remote civilian hospitals without an on-site radiologist the same ability to provide professional interpretations of exams for their patients, thereby minimizing unnecessary investigations and medical evacuations. In the case of battlefield teleradiology, it enables specialized radiologist interpretation of studies performed as close to the point of injury as possible, even if a radiologist cannot be on site where the images were acquired. Teleradiology has demonstrated itself to be a force multiplying application of telemedicine on the battlefield; however, achieving standard of care in this capacity for our deployed force requires significant logistical support that is easily overlooked or underestimated. 


\section{Historical Context}

\section{Civilian Teleradiology}

The earliest form of civilian teleradiology in the US predates the modern computer and digital imaging. Closed-circuit television was the initial medium used to transmit medical images, including radiographs, between remote locations in the mid-1960s. Dr. Kenneth Bird created an interactive television via a microwave link that enabled telemedicine consultations from Massachusetts General Hospital to nearby Logan Airport and other medical facilities within Boston [1]. At the time, radiologic images were just a small component of the larger telemedicine system that included other fields with easily broadcast data such as dermatology and pathology. Walter Reed General Hospital (later renamed Walter Reed National Military Medical Center) also adopted the television to transmit radiographs between the emergency and radiology departments [2]. Conventional radiographs were transmitted in realtime to the interpreting radiologist, but diagnostic capability was significantly limited by poor spatial and contrast resolution. Additionally, the television system was expensive to install and carried cumbersome logistical requirements [2]. These limitations of television as a teleradiology medium were compounded by the later introduction of crosssectional imaging including CT and US with larger sets of data. By the 1970s, television teleradiology was relegated to novelty status and had fallen out of clinical practice.

A computer-based approach to teleradiology gained momentum in the late 1970s and 1980s, forming the foundation for modern teleradiology. This system utilized a "store and forward" method whereby images were acquired in analog format, digitized and stored on a computer, then transmitted via telephone connection to an offsite radiologist for interpretation. Unlike with television, this computer-based process decoupled the acquisition, transmission, and interpretation phases of teleradiology. This made teleradiology more practical because now the patient, radiologist, and support staff did not have to be present at both sites simultaneously [2].

One of the first well-documented commercial applications of digital teleradiology in the 1980s involved after-hours coverage of an emergency department in New York City by residents at a larger nearby academic facility [3]. Due to low bandwidth inherent to telephonic transmission at the time, $\mathrm{CT}$ and radiographic films required significant degradation of quality in order to achieve transmission times shorter than $10 \mathrm{~min}$ per exam. These digitized exams were interpreted by the teleradiologist on an 11-in. computer monitor fitted with rudimentary digital zooming and image manipulation capabilities. Surprisingly, inadequate image quality contributed to only $1.6 \%$ of the clinically significant interpretation discrepancies when compared to direct interpretation of the original hardcopy films [3]. Digital teleradiology was also hindered by challenges in communicating patient information pertinent to the images. For example, the patient's medical history, indications for the exam, and the final interpretation for each study had to be communicated separately by telephone or fax [4].

Dramatic advances in computer technology and telecommunications in the 1990s addressed many of these technical challenges, ushering in a new era for civilian teleradiology in the US. Computer platforms became faster and more affordable. Direct digital acquisition of radiology studies improved image quality and eliminated resources previously required for analog to digital conversion. High bandwidth internet expanded inter-facility connectivity and decreased transmission times. Widespread adoption and integration of picture archiving and communication systems (PACS) and radiology information systems (RIS) streamlined retrieval of clinical data and eased communication between ordering providers and interpreting radiologists [4]. By 1997, over $50 \%$ of radiology practices in the US utilized teleradiology in one form or another [5]. Teleradiology enabled smaller or rural hospitals without night-time in-house radiologists to maintain highquality interpretations around the clock, while also augmenting day-time coverage during periods of increased imaging volume or on-site radiologist absence, mitigating the need for more expensive on-site solutions. The practice of teleradiology in the US was further legitimized when the American College of Radiology Task Force published standards for practice in 1994 and later refreshed them in 2005 and 2012. Civilian teleradiology has also differentially succeeded in the US over other telemedicine services because of its consistent reimbursement by Medicare and other health insurance agencies, which pay the same rate as in-person physician services $[6 \bullet \bullet$.

\section{Military Teleradiology}

The success of civilian teleradiology in the 1990s was bolstered by a parallel and collaborative effort by the US Department of Defense (DOD). Facing enormous technologic and financial challenges in caring for its highly mobile and frequently disconnected population, the military hoped that telemedicine would improve access to and continuity of care, as well as reduce long-term healthcare costs.

Following several years of research and development at the University of California Los Angeles (UCLA), the US Army Medical Research and Materiel Command (USAMRMC) funded the first large-scale implementation of a standardized PACS in several of its major domestic and foreign medical centers in 1991, allowing for digital storage and transmission of radiology examinations [7]. Madigan Army Medical Center in Tacoma, Washington, was among the first three of the tertiary care military treatment facilities to implement PACS. The DOD hoped that using a standardized PACS would allow for a permanent, mobile imaging record for its patients. PACS 
technology would also facilitate the electronic storage and transmission of CT exams in a deployed environment.

The USAMRMC developed a battlefield CT scanner for use in a deployed military hospital in 1986. Each scanner was housed in a modular hardwall shelter (ISO-shelter) similar to that of which combat hospitals of the time were built (Fig. 1). Prior to deployment, these CT scanners underwent clinical testing at Brooke and Fitzsimons Army Medical Centers in 1990. More than 1000 patients were scanned at the two sites, including trauma patients whose injuries resembled those seen in combat [8]. This testing in the US demonstrated the feasibility of deploying a CT scanner on the battlefield.

In 1991, CT scanners were deployed in support of the First Gulf War, Operation Desert Storm, as part of the US Army's 86th and 12th Evacuation Hospitals located in the northern Saudi Arabian desert approximately 50 miles from the Iraqi border [8]. CT imaging proved to be a successful triage modality, helping to determine which patients needed emergency surgery and which could be evacuated to a higher level of care. Physicians were able to make diagnoses that had rarely been possible before under battlefield conditions with plain radiography. Typical of most wars, the majority of the patients treated were categorized with diseases and non-battle injuries, such as polycystic kidney disease and appendicitis. Actual battle-related injuries at which $\mathrm{CT}$ scans excelled included those related to internal visceral organ trauma from blunt force injury, or complex neurologic or musculoskeletal injuries, such as lumbar vertebral burst fractures with spinal canal compromise. The usefulness of CT during this conflict led to its subsequent inclusion as standard equipment for all future combat support hospitals in the US Army [9].

The First Gulf War was also the first time battlefield teleradiology was tested in war (Fig. 2). A teleradiology link utilizing satellite communications was established with Brooke Army Medical Center, allowing a total of $41 \mathrm{CT}$ exams to be reviewed for second opinion by subspecialty trained radiologists [8]. This teleradiology component was primarily for testing purposes. It was not a requirement for achieving standard of care, because both evacuation hospitals had experienced radiologists on site. Furthermore, both the radiologists and technologists who ran the equipment were already familiar with it due to its extensive predeployment testing in San Antonio, Texas. Physical installation and initial maintenance of the CT equipment were also provided by manufacturer representatives, who deployed with the hospitals [8].

After Operation Desert Storm demonstrated the potential of teleradiology, the DOD began utilizing this technology across other continents. Teleradiology was implemented on the Korean peninsula between 1993 and 1994. Four military radiologists were tasked with providing all radiologic coverage in South Korea, encompassing approximately 40,000 troops and military dependents. Prior to teleradiology, radiology studies could take up to 2 weeks to be interpreted due to lengthy transportation times. This process often delayed care and caused unnecessary medical evacuations. These problems were reduced and teleradiology flourished in South Korea.

Fig. 1 International organization for Standardization Shelter (ISOShelter) used to house radiology equipment as part of a Combat Support Hospital (CSH)

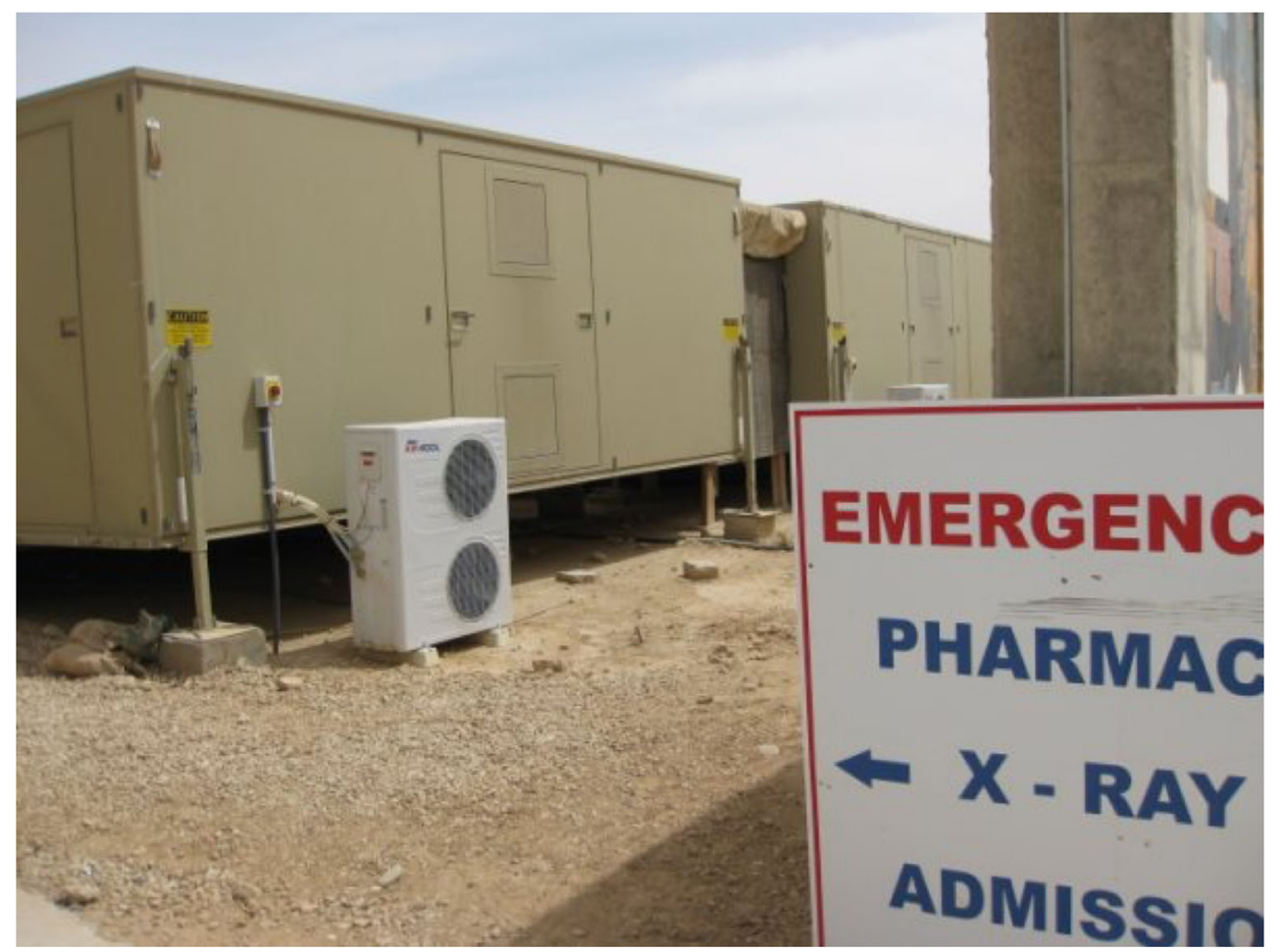


Fig. 2 Radiology network enabling military radiologists stationed at large medical centers in the US and Europe to provide teleradiology support to forward deployed combat support hospitals

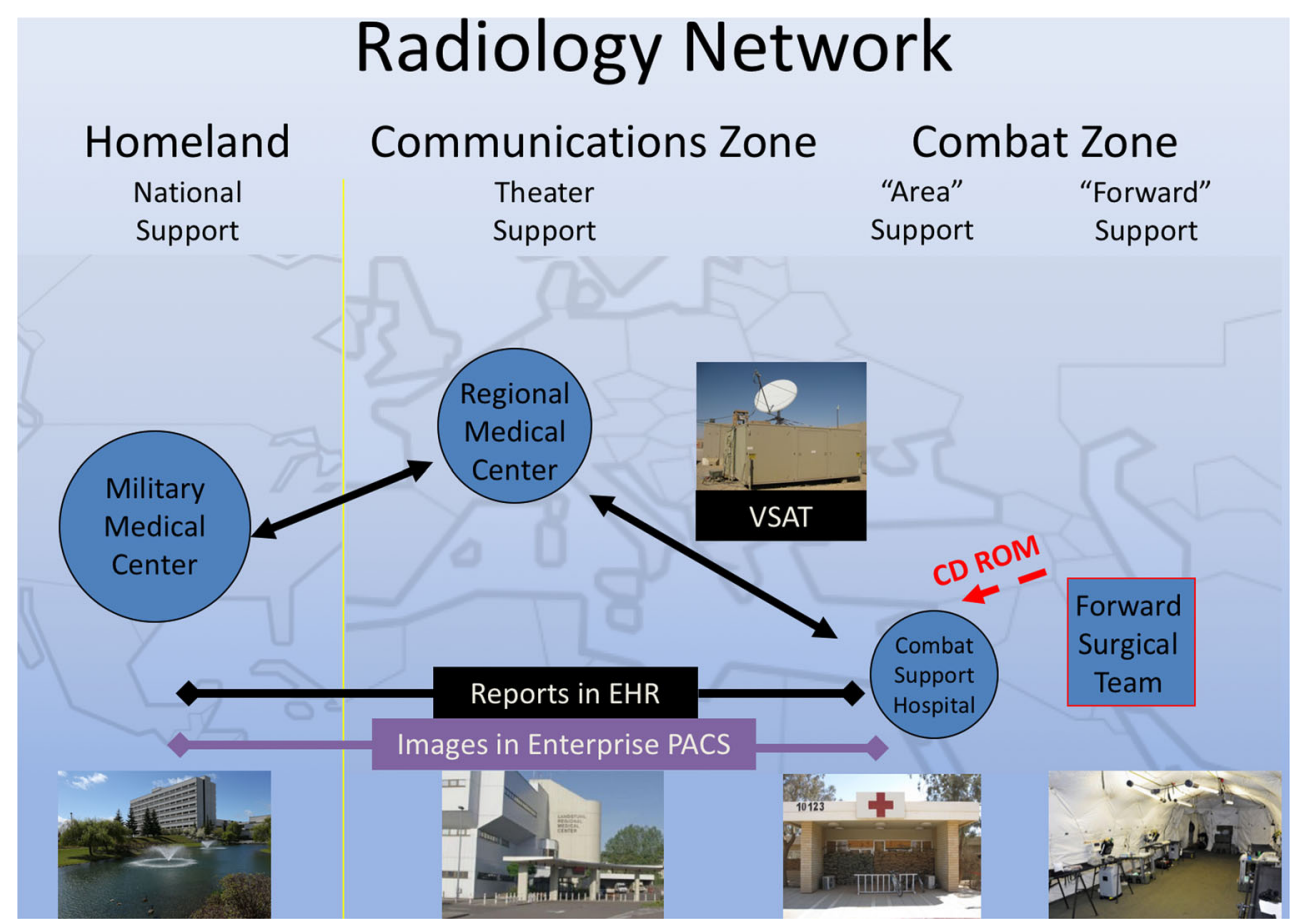

In 1995, several combat hospitals with embedded CT scanners were deployed to the Bosnia region as part of the NATO's peacekeeping operation [9]. Unlike the first Gulf War, there was not a radiologist at every hospital with a CT scanner. A radiologist was deployed at the hospital in Hungary, which was over 100 miles away from the forward hospital in Bosnia. Radiologist's support was to be provided via teleradiology capability. Teleradiology became essential for limiting unnecessary and potentially dangerous evacuations. To evacuate a casualty by ground to a higher level of care required at least four vehicles and eight combat soldiers. Likewise, evacuation by air required two or more helicopters, including an attack helicopter to serve as a security escort. A teleradiology link was utilized from the hospital in Bosnia to transmit X-ray, ultrasound, and CT exams to an American radiologist in Hungary or Germany. Over 10,000 exams were interpreted remotely in this manner over the course of the conflict, the first time that teleradiology managed the entire workload of a combat hospital without a radiologist on site [9].

The success of teleradiology in Bosnia was due in large part to the overwhelming logistical support with which it came. The CT scanner and teleradiology system received around-the-clock support by a group of civilian engineers and technicians from Georgetown University, who were responsible for the initial installation and maintenance through the deployment. Maintenance was carried out by on-site technicians and remote technical support. There were also standard operating protocols established and followed for the acquisition, transmission, and interpretation of radiology exams [9]. Despite these successes, the lack of an on-site radiologist may have contributed to challenges in image quality, appropriate utilization, and radiation dose management.

The CT scanner and teleradiology have continued to play a major role during more recent combat operations in Afghanistan and Iraq. Battlefield mortality has been reduced by pushing surgical assets forward as close as possible to the point of injury in the form of forward surgical teams (FSTs). At the beginning of the operations in Iraq and Afghanistan, these FSTs were typically only equipped with x-ray, fluoroscopy, and ultrasound, which were used for point of care decisions, and were not interpreted in real-time by a radiologist. Examples include chest X-rays to determine appropriate endotracheal intubation, fluoroscopy in support of orthopedic fixation, or Focused Assessment with Sonography in Trauma (FAST) exams to guide initial triage. However, these conventional modalities do not replace the CT scanner in trauma.

Just as it has in the civilian world, CT imaging has evolved to become standard of care for initial triage, therapy planning, and follow-up evaluation of trauma patients on the battlefield. In 2002, during Operation Enduring Freedom, CT was utilized in Bagram, Afghanistan, to triage and manage complex craniofacial trauma patients [10]. The extent of injury for many of these patients could not have been determined without a CT scan. With no neurosurgeon on site, CT scans were used to determine if patients required immediate evacuation to a higher level of care.

For these reasons, and due to the nature of the modern non-linear battlefield with a requirement for wide area support, CT scanners have increasingly been pushed 
outward to smaller-sized medical units in support of splitbase operations. In this model, the resources of one combat support hospital (CSH) may be deployed as three small hospitals with a wide geographic area of responsibility (Fig. 3). While a typical CSH may have one or two radiologists, each of these three smaller hospitals would typically have a CT scanner. This results in CT scanners being deployed without a collocated radiologist. Teleradiology has been leveraged to create staffing efficiencies in support of split-base operations, but determining minimum acceptable staffing levels requires judgment and the balancing of risk. The role of the radiologist as a leader in quality, safety, efficiency, and appropriate utilization should be considered particularly when CT scanners, a significant source of radiation exposure with complex maintenance requirements, are included in the deployment package.

Severely injured patients are rapidly transported through the echelons of care from point of injury to the FST, to the CSH, then to the theater support hospital (e.g., Landstuhl Regional Medical Center in Germany), and finally back to the USA (Fig. 2). A networked electronic medical record and enterprise PACS also permit the transmission of radiology information rapidly throughout these levels of care starting at the CSH. This process dramatically increases the efficiency of care and mitigates the need for repeating many radiology studies at each site.

\section{Challenges and Lessons Learned}

The standard of care for battlefield teleradiology, like all other aspects of patient care, is no different from the civilian standard upheld in the US. By doctrine, limited resources encountered in a deployed environment do not change the standard of care, and when resources are exceeded the patient should be evacuated. Only when a servicemember's life, limb or eyesight are at immediate and undeniable risk does the concept of field expediency allow for providing temporary treatment below that of the established standard of care. Fortunately, these situations are typically uncommon in a modern deployed setting. When radiology assets are forward deployed with minimal staffing, there is increased risk of inappropriate utilization, poor image quality, and radiation over-exposure that must be addressed according to the standard of care. Meeting standard of care with battlefield teleradiology can be a difficult task that must address equipment, maintenance, technologist, and physician radiologist staff requirements.

It is easy to forget that the radiologist's interpretation is just one step in the imaging process, and teleradiology addresses only this final step. Pre-interpretive and post-interpretive radiologist supervision is required as are specialized PACS and Information Technology (IT) support personnel. A portion of the radiology oversight role is centrally located at Landstuhl Regional Medical Center (LRMC) in Germany, a hub of

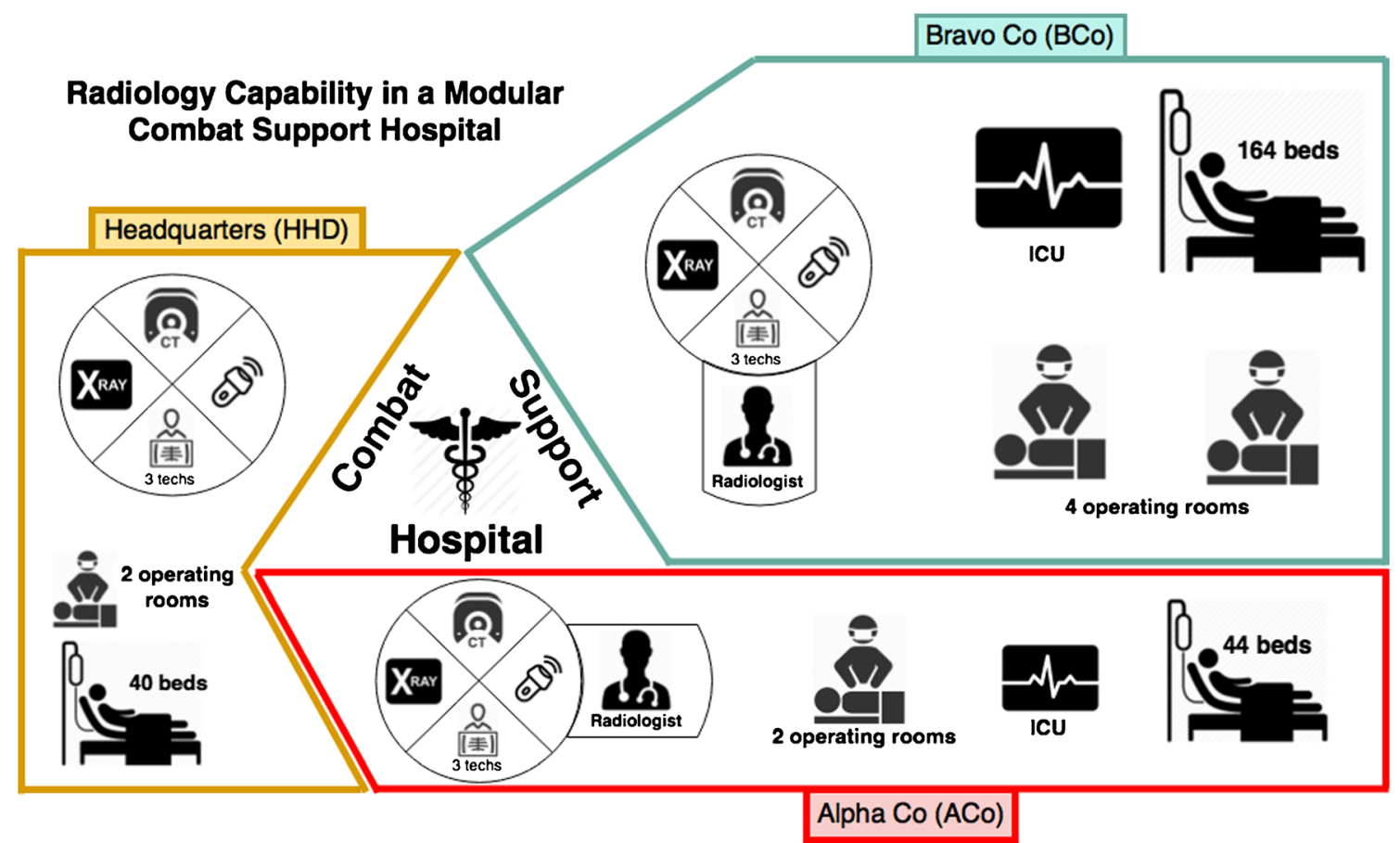

Fig. 3 Radiology Capability in a Modular Combat Support Hospital (CSH). A standard CSH is composed of three modular components, including Headquarters and Headquarters Detachment (HHD), Alpha Company ( $\mathrm{ACo}$ ), and Bravo Company (BCo). Depending on operational requirements, a CSH may be deployed as single unit or as three small hospitals during split-base operations, with each small hospital covering a wide geographic area of responsibility. Each small hospital can have CT, X-ray, and ultrasound capability; however, only one or two sites will have an on-site radiologist 
Fig. 4 Computed tomography (CT) scanner housed in an ISOShelter with multiple airconditioning units to prevent equipment over-heating, a commonly encountered environmental challenge in the deployed setting. Other countermeasures include deploying sunshades over the CT scanner ISO-shelter, improvising air filters placed on ventilation ducts to protect against dust infiltration, and employing uninterruptable power source to protect against power fluctuations

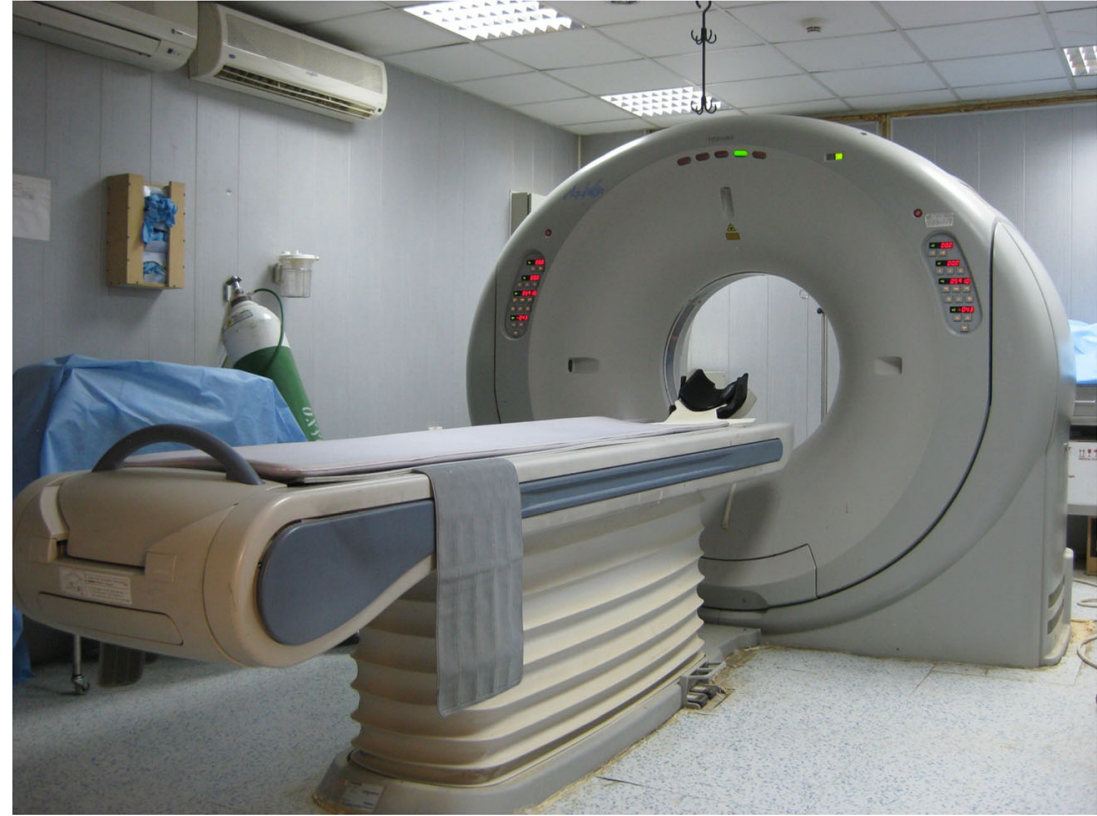

casualty evacuation from conflicts in Iraq and Afghanistan. This oversight requires significant additional PACS personnel and radiologists with specific skillsets to oversee interpretive and non-interpretive factors in radiology.

Serviceable equipment and qualified operators are also required. The importance of properly training technologists and other ancillary staff that perform and deliver the images to the radiologist cannot be understated. Poor quality images have a significant negative impact on the ability of the radiologist and treating clinician to interpret the image and manage patients. Further, this problem is not unique to the military.

Medecins Sans Frontiers (MSF) provides teleradiology coverage for remote facilities with X-ray capability within Sub-Saharan Africa, Central Asia, and Southeast Asia. A retrospective review of all $\mathrm{X}$-rays interpreted via teleradiology in 2012 showed that the percentage of non-diagnostic X-rays produced across these facilities ranged from $11 \%$ to as high as $87 \%$ [11•]. Not surprisingly, the lowest performing sites for quality were those for which experienced MSF radiographers had not provided initial installation, training, or regular on-site quality assurance. Not only did this study underscore the importance of technologist training but it also suggested that these remote sites without properly trained personnel and quality assurance programs were detrimental to patient care and should be discontinued [11•]. The challenges faced by MSF in meeting standard of care have resembled those encountered in battlefield teleradiology. Furthermore, the challenge increases with more sophisticated capabilities such as $\mathrm{CT}$ and magnetic resonance imaging (MRI).

A case study of the rapid fielding of MRI in Afghanistan in 2012 underscores the importance of planning and support to ensure success. Imaging patients in theatre with mild traumatic brain injuries (m-TBI), spinal trauma, and musculoskeletal injuries enabled clinicians to avoid potentially costly and unnecessary evacuations to LRMC in Germany. Some of the Brain MRIs were interpreted remotely by specialists using teleradiology. Performing MRI in an austere environment such as Afghanistan represents a significant technological achievement and should serve as a proof in concept. However, the volume of MRIs performed in Afghanistan was rather small as it was challenged by issues related to infrastructure, supply lines, and staffing. Future fielding of MRI in conflict zones would benefit from increased radiologist leadership beyond the interpretive tasks of teleradiology typically thought of by non-radiologist planners.

Radiology technologists are an essential component of the radiology process along with the imaging hardware, maintenance support, and the interpreting radiologist. Technologist training and experience are important factors to consider when including radiology capabilities in a deployed hospital. There are certifying institutions for X-ray, CT, Ultrasound, and MRI that ensure technologists have the proper education and demonstrate subject matter expertise. For example, standard of care requires Registry certification. Civilian trauma centers mandate CT technologists to pass a Registry Exam that requires significant training and study to pass. Because CT is a relatively new addition to battlefield medicine, it is important to ensure that X-ray technologists are CT registry certified if they will be expected to perform CT. A short course of training on basic $\mathrm{CT}$ functions is insufficient when imaging complex war trauma, and it is not recommended.

There are multiple responsibilities of the radiologist in addition to the interpretation of images, and many of these responsibilities are difficult to address by teleradiology. For example, it is the responsibility of the physician radiologist to ensure proper radiation dose, scan protocol development and 
selection, and indication for exam. In the setting of teleradiology, the off-site radiologist will likely only be able to address these issues retrospectively as they will only become aware of the problem when they are reviewing the images. Radiologists are also the physicians most familiar with recognizing and managing the spectrum of reactions to iodinated and gadolinium-based contrast; therefore, not having one physically available may be a detriment to patient care.

The next link in the radiology process is maintenance support. Equipment must be properly maintained to meet standard of care. Maintenance of CT, X-ray, and ultrasound equipment in the deployed environment is challenging. Environmental extremes of heat, cold, moisture, dust, and power fluctuations take a toll on the delicate equipment utilized in radiology. These factors may lead to significant down time that can be troublesome during military operations. Familiarity with the equipment and the environment are necessary when implementing countermeasures. For example, regular vacuuming and improvised air filters placed on ventilation ducts have been employed with some success [12]. Additional airconditioning units, sunshades deployed over the building housing a CT scanner, and an uninterruptable power source (UPS) large enough to protect a modern CT or MRI scanner are other countermeasures that have been utilized (Fig. 4). A team of maintenance technicians familiar with vendor specific hardware is essential to reliable radiology services. A technician who is familiar with a particular system can provide organization-level maintenance, but intermediate-level maintenance may require a vendor contractor. Unless such a vendor contractor is available onsite, it may take a week or more (if it is possible at all) to fly one in to service the equipment. Such interruptions in radiology services may be unacceptable.

The computer and telecommunications requirements to conduct teleradiology are significant. Most teleradiology in the US is conducted over fiberoptic networks which are not available in the deployed environment. Satellite links are utilized, but such communications can be intermittent and slow unless sufficient bandwidth is dedicated for radiology purposes. Other limitations of satellite communication include cost, significant contractor support requirements, potential weather impacts (a satellite does not work well or at all during sandstorms), and a lack of redundancy. If images cannot be transmitted from the remote site of acquisition to the radiologist, then teleradiology does not work.

The ability of teleradiology to conduct continuous operations $24 \mathrm{~h}$ daily is determined by the reliability of its personnel, equipment, and communications. Sustaining the workforce is critical to conducting continuous operations. Radiology is typically one of the busiest services in the hospital, and appropriate staffing levels should be considered. Teleradiology has been used successfully to augment physician radiologist coverage within a split-base CSH by sharing on-call responsibilities. One radiologist in the CSH would cover all three sites overnight by using teleradiology, allowing the other radiologists to perform non-interpretive duties or to institute a sustainable work-rest cycle.

\section{Conclusions}

Radiology is an essential component of medical care on the battlefield, and its role has expanded with the introduction of CT scanners deployed forward as close to the point of injury as possible. Teleradiology enables flexible staffing models to ensure sustainable physician coverage, but image interpretation is only one link in the imaging chain, and it is not a substitute for actively engaged radiologist leadership on the ground wherever a CT scanner is located.

Acknowledgments The authors would like to acknowledge the manuscript review and commentary from Justin Dodge, M.D.

\section{Compliance with Ethical Standards}

Conflict of Interest Drs. Bess and Roberge declare no conflicts of interest relevant to this manuscript.

Human and Animal Rights and Informed Consent This article does not contain any studies with human or animal subjects performed by any of the authors.

\section{References}

Papers of particular interest, published recently, have been highlighted as:

- Of importance

•- Of major importance

1. Bird KT. Cardiopulmonary frontiers: quality health care via interactive television. Chest. 1972;61(3):204-5.

2. Thrall JH. Teleradiology. Part I. History and clinical applications. Radiology. 2007;243(3):613-7.

3. Kagetsu NJ, Zulauf DR, Ablow RC. Clinical trial of digital teleradiology in the practice of emergency room radiology. Radiology. 1987;165(2):551-4.

4. Thrall JH. Reinventing radiology in the digital age. Part II. New directions and new stakeholder value. Radiology. 2005;237(1):158.

5. Boland GW. Teleradiology: another revolution in radiology? Clin Radiol. 1998;53(8):547-53.

6.• Silva III E, Breslau J, Barr RM, Liebscher LA, Bohl M, Hoffman T, et al. ACR white paper on teleradiology practice: a report from the Task Force on Teleradiology Practice. J Am Coll Radiol. 2013;10(8):575-85. doi:10.1016/j.jacr.2013.03.018. American College of Radiology Report that describes the current state of civilian teleradiology and provides comprehensive best- 
practice guidelines for its continued practice in the United States.

7. Mogel GT. The role of the Department of Defense in PACS and telemedicine research and development. Comput Med Imaging Graph. 2003;27(2-3):129-35.

8. Cawthon MA, Goeringer F, Telepak RJ, Burton BS, Pupa SH, Willis CE, et al. Preliminary assessment of computed tomography and satellite teleradiology from Operation Desert Storm. Invest Radiol. 1991;26(10):854-7.

9. Mun SK, Levine B, Cleary K, Dai H. Deployable teleradiology and telemedicine for the US military. Comput Methods Programs Biomed. 1998;57(1-2):21-7.

10. Statler JD, Tempel CG, Harcke HT. Computed tomography of craniofacial trauma at a combat support hospital in Afghanistan. Mil Med. 2005;170(3):206-10.
11. Spijker S, Andronikou S, Kosack C, Wootton R, Bonnet M, Lemmens N. Quality assessment of X-rays interpreted via teleradiology for Médecins Sans Frontières. J Telemed Telecare. 2014;20(2):82-8. doi:10.1177/1357633X14524153. Retrospective study by an international civilian teleradiology practice that examines the diagnostic quality of $\mathrm{X}$-rays produced at remote sites by technologists with a variable degree of training. This study demonstrates the importance of having properly trained technologist at sites utilized for teleradiology in order to meet standard of care.

12. Harcke HT, Statler JD, Montilla J. Radiology in a hostile environment: experience in Afghanistan. Mil Med. 2006;171(3):194-9. 